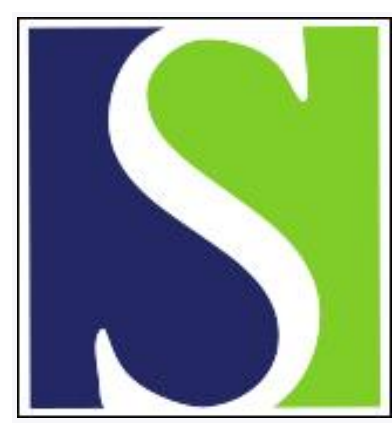

Scand J Work Environ Health 1990;16(3):153-157

https://doi.org/10.5271/sjweh.1801

Issue date: 01 Jun 1990

Interpretation of "negative" studies in occupational epidemiology.

by Ahlbom A, Axelson O, Stottrup Hansen ES, Hogstedt C, Jensen UJ, Olsen J

Affiliation: Department of Epidemiology, Karolinska Institute, Stockholm, Sweden.

This article in PubMed: www.ncbi.nlm.nih.gov/pubmed/2143311

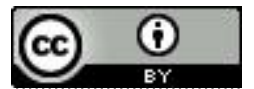




\title{
Interpretation of "negative" studies in occupational epidemiology
}

\author{
by Anders Ahlbom, PhD, ${ }^{1}$ Olav Axelson, MD, ${ }^{2}$ Eva Støttrup Hansen, MD, ${ }^{3}$ \\ Christer Hogstedt, MD, ${ }^{4}$ Uffe J Jensen, PhD, ${ }^{5}$ Jørn Olsen, $\mathrm{MD}^{6}$
}

\begin{abstract}
AHLBOM A, AXELSON O, STøTTRUP HANSEN E, HOGSTEDT C, JENSEN UJ, OLSEN J. Interpretation of "negative" studies in occupational epidemiology. Scand J Work Environ Health 1990; 16:153-7. Two criteria for an interpretation of noneffect are that the relative risk estimate be near unity and that the confidence interval be narrow; lack of statistical significance has no bearing on this issue. A further requirement is that the relative risk estimate not be near the null value as an effect of nondifferential exposure misclassification, negative confounding, or some other systematic error. Occasionally, when results are unexpected or difficult to accept, studies are "underinterpreted" as negative or inconclusive on the basis of arguments such as "confounding," "crude exposure assessment," or "lack of a known mechanism." The present paper supports the position that these arguments are commonly invalid. Scientific standards should be used to separate causal associations from noncausal ones, but in public health decisions this practice has to be balanced by the principle that the "benefit of the doubt" should be given to the persons subject to potential risk.
\end{abstract}

Key terms: epidemiologic methods, negative results, occupational epidemiology, precision, validity.

Epidemiologic studies have an important role in the identification and evaluation of potential risk factors in the work environment. Studies indicating that some agent or process in the work environment might increase the risk of disease are thoroughly scrutinized. Certain criteria for this scrutiny have even been suggested, the most well-known being those by Hill (1).

It should, however, be of equal importance to evaluate those studies which do not suggest any effects of a particular exposure on the risk of disease, ie, studies in which the effect measure is estimated to be near the null value. This problem has been discussed by Hernberg (2) and recently in an editorial in the New England Journal of Medicine (3).

Studies with an effect estimate near the null value fall roughly into two categories. First, such a study may actually suggest that the studied exposure lacks effect on the disease risk, although this suggestion may be weak if the study size is limited. Indeed, if the size is

1 Department of Epidemiology, Institute of Environmental Medicine, Karolinska Institute, Stockholm, Sweden.

1 Department of Occupational Medicine, Linköping University, Linköping, Sweden.

3 Department of Social Medicine, Institute of Community Health, Odense University, Odense, Denmark.

${ }_{4}$ Departments of Occupational Medicine, Karolinska Hospital and National Institute of Occupational Medicine, Stockholm, Sweden.

5 Department of Philosophy, University of Århus, Århus, Denmark.

6 Department of Social Medicine, University of Ảrhus, Århus, Denmark.

Reprint requests to: Professor A Ahlbom, Department of Epidemiology, Institute of Environmental Medicine, Karolinska Institute, Box 60208, S-104 01 Stockholm, Sweden. small, the study may be virtually void of information. Second, a study may not indicate an effect because of systematic errors which bias the effect measure towards its null value. In such instances the results are also virtually inconclusive but may be misleading to the reader who does not appreciate the problems of the study. One objective of the present article was to analyze the basis for distinguishing between these situations. There are studies which are being "underinterpreted" as indicative of no effect or as inconclusive, despite the fact that they do suggest an effect of the exposure. A further objective of this article was to scrutinize some of the arguments being used when study results are underinterpreted. The strengths and limitations of combining the results of several studies in a meta-analysis are also discussed. Some public health aspects and related decisions regarding preventive actions are discussed in a concluding section.

This article is a synthesis of a more comprehensive report (4).

\section{No effect or no information}

According to Popper (5), hypotheses may be rejected but not proved. Applied to epidemiology, this assumption would mean that the hypothesis of no effect, of an exposure on the risk of a disease, may be rejected, but never proved. The strongest negative conclusion would be that there are no grounds for rejection. In other words, it might be proved that an exposure has an effect, at least in one population, but not that it generally lacks effect. There is no logical procedure with which to draw universal conclusions from a 
limited domain of data. Due to the presence or absence of synergistic factors, an exposure may have no impact in a studied population but may well have an effect on other populations. This strict application of the Popperian philosophy fails, however, to meet the requirements of epidemiology, particularly in regard to public health actions (6). Instead, in the spirit of what may be referred to as inductivism, an epidemiologic study is considered a measurement exercise, with which relevant measures of effect are estimated (7). The parameter value corresponding to no effect, the null value, is considered only one value among others. The best possible conclusion is drawn on the basis of all relevant information available at the time. In this process, any investigation that indicates that the exposure does not increase the risk of disease in a studied population corroborates the hypothesis of no effect.

This view provides the basis for the subsequent discussion on no effect or no information.

\section{Random errors}

If the size of a study is small, the study becomes susceptible to random errors. Generally the number of exposed cases is the critical number. A small study results in a low precision in the estimate of the effect measure. In such instances there is no justification in concluding that the effect measure is close to the null value merely because the estimate is.

Significance testing has been the traditional method when randomness is evaluated in epidemiologic data. This procedure is inappropriate, however. It arbitrarily dichotomizes study results into significant and nonsignificant results. Although perhaps convenient, this dichotomy means that useful information from the study is neglected, and it forces studies with probability values (P-values) of, say, 0.049 and 0.051 , to fall into opposite categories. When the result of a study is pooled with other relevant information, one should use as much of the information in the study as possible and not only whether the P-value exceeds 0.05 .

The use of the actual P-value, rather than the common dichotomy, would not be appropriate either. The $P$-value depends on the magnitude of the estimated effect parameter but also, in an inseparable way, on the study size. Therefore, the same P-value may correspond to different effect parameter estimates, depending on the size of the study.

The major limitation of the P-value, however, stems from the fact that it is calculated on the condition of the null hypothesis, ie, on the assumption that the null value is true. Hence, it only provides information on the consistency between the observed data and the null hypothesis, but not on the consistency with other hypotheses. Therefore when the $\mathrm{P}$-value is high, one does not know for which range of values of a parameter the P-value is also high, and one does not know for which values of the parameter the P-value is low. This situation is always a severe limitation, but it is perhaps even more important for the studies under consideration in this article, ie, studies indicating no effects. Specifically, the fact that a P-value exceeds 0.05 has no bearing on the issue of whether or not the effect parameter is equal to the null value. This same statement can be made when the P-value is supported by a high power (probability of a low P-value) (8). A series of P-values corresponding to different hypotheses about the effect mieasure (the P-value function) would not provide the missing information, but this information is generally not useful for publications. This information, however, is also summarized by the confidence interval, and the confidence interval should be used to report the precision of a study $(9,10)$. Thus, in order for a study to indicate that an exposure has no effect on the risk of disease, it is necessary that the confidence interval be narrow and located around the null value.

\section{Systematic errors}

A further requirement for interpreting a study as indicating no effect is that the effect parameter not be near its null value as a consequence of systematic errors. Such errors fall into two categories, those which will always push the effect estimate towards the null value and thereby lead to an underestimation of the effect, if any, and those which, depending on the circumstances, may lead to an over- or underestimation of an effect.

One major element in this first category is the occurrence of those instances in which the difference in the level of exposure between the contrasted groups appears greater than it actually is, for instance; in situations in which subjects are assigned to the wrong exposure categories with the same probabilities regardless of whether they develop the disease or not (nondifferential misclassification of exposure). The result will always be dilution, and the effect estimate will be pulled towards its null value (8). Hence, a study can never be taken as an indication that the exposure is lacking effect if the exposure assessment has been inflicted with nondifferential misclassification.

The nondifferential misclassification of exposure comes in several different shapes. First, it may arise whenever exposure is assessed by means of an uncertain measurement procedure, ie, whenever the measurements themselves are performed with a certain randomness. For this reason, the nondifferential misclassification of exposure is sometimes referred to as random misclassification. Second, however, it also appears when a proxy with limited correlation to the actual exposure, such as job title, is being used to assign exposure categories for the subjects in a study. Third, a misconception of the biologically relevant exposure may result in nondifferential exposure misclassification. One example would be the use of a point-in-time exposure intensity rather than the cumulative dose. Whenever the correlation between the intensity and the 
cumulative dose is low, the exposure contrast will appear to be greater than it actually is, and the effect, if any, will be underestimated. Erroneous assumptions about induction time is another example leading to the same effect.

It is worth noting that one consequence of the aforementioned problems is that an existing dose-effect relationship may level out. Thus failure to demonstrate an effect gradient is not generally a strong indicator that the exposure lacks effect on the risk of disease.

Diagnostic misclassification with the same sensitivity and specificity regardless of exposure status is referred to as nondifferential. Low specificity leads to the inclusion of subjects without the disease among the cases. This situation gives rise to dilution just as did the exposure misclassification that has already been discussed and, hence, also to an underestimation of the effect, if any. This underestimation of the effect also derives from situations in which several diagnostic categories are collapsed into one group, as when all leukemia is used as the outcome instead of, eg, acute myeloid leukemia. Thus one cannot conclude from an analysis of all leukemias taken together that an exposure has no effect on acute myeloid leukemia.

The second category of systematic errors consists of those which, depending on the circumstances, lead either to an over- or underestimation of the effect. The relevant circumstances are the ones which lead to underestimation. One such situation is negative confounding. This type of confounding occurs whenever a risk factor, other than the exposure under study, is more prevalent among the unexposed than among the exposed. The healthy worker effect may, in some instances, be taken as one example of this circumstance with specific relevance to occupational epidemiology (11). When specific occupational groups are compared to the general population, or to defined segments of that population, the total mortality and the mortality of several major causes of death are consistently found to be lower in the occupational group. This occurrence could mask an effect of the exposure.

Specific risk factors of the disease could also create negative confounding. However, epidemiologic theory indicates that, in order for a confounder to produce a sizeable bias, both the association with the exposure and the association with the disease have to be substantial and at least one of them has to be very strong (12).

Differential diagnostic misclassification, and differential exposure misclassification as well, can create systematic errors leading to an underestimation of the effect parameter. One example would be a study in which the information underlying the diagnosis is reviewed carefully for the exposed cases but not for the unexposed cases, eg, if incidence rates in a specific cohort are compared to reference rates taken from, eg, a cancer registry. Another example could be instances in which workmates or spouses of deceased cases in a case-referent study give less accurate exposure information than living referents.

Each epidemiologic study is based on a specified population observed for the occurrence of disease during a specified period of time, the so-called study base (13). In cohort studies the entire study base should, in principle, be observed, but in practice subjects are lost due to nonresponse, unknown location, wrong identification code, and so forth. If this is the situation more often for exposed cases than for others, ie, if the selection procedure is related both to exposure and outcome, a selection bias would occur which would lead to an underestimation of the effect parameter.

In case-referent studies, the study base is represented by a sample. An inappropriate sampling procedure may lead to selection bias, which could result in the masking of an effect. One example of such a situation could be the use of hospital referents with diseases for which the exposure under study is a risk factor. The proportion of exposed subjects in this reference group would then be expected to be higher than in the entire study base, and a possible effect would be underestimated.

\section{Combination of data from several studies}

When several studies exist, the evaluation might be improved if their results are combined. When data are formally pooled into one single analysis, it is called a meta-analysis (14). One objection to this procedure has been the so-called "publication bias." This term refers to the notion that studies not showing increased risks are less likely to get published, especially, perhaps, when the hypothesis is new and somewhat vague. This, however, is merely speculation, and we have been unable to find much information supporting this notion, although some arguments in favor of the existence of publication bias have been provided (15). On the other hand there seem to be valid arguments for deeming this problem to be small $(3,4)$.

Several statistical methods for combining study results in a pooled result are available (6). The aim of the meta-analysis is to use the total amount of information. The techniques available for meta-analysis are well suited to taking the varying degree of precision in the different studies into consideration. The problem remains, however, that the pooled studies will also vary with regard to study base, study design, and validity, variations which the meta-analysis does not take into account. Hence, the meta-analysis must be applied with great caution.

\section{Underinterpretation of epidemiologic results}

As was mentioned in the Introduction, epidemiologic results are occasionally interpreted as showing no effect 
of the exposure although a closer look at the design and results of the study might lead to the conclusion that the study is inconclusive. One also encounters studies which have been interpreted as inconclusive despite the fact that they show clear effects and apparently are not invalidated by systematic errors which might lead to overestimation. Such "underinterpretations" have occurred when the results have been unexpected or for other reasons difficult to accept.

\section{Lack of statistical significance}

If the observed data in a study show a considerably increased risk for the exposed subjects, but the confidence interval includes the null value, the interpretation may sometimes be that the true value equals the null value. For reasons that have already been discussed, this is an incorrect interpretation. Despite the fact that there is no "statistical significance," this study lends support to the hypothesis that there is in fact an increased risk for the exposed. The support tends to be weak, however, since the precision of the study is limited. This weak precision would become clear in the aforementioned meta-analysis, in which the weight assigned to such a study would be correspondingly low.

\section{Poor exposure assessment}

Sometimes studies showing associations between exposure and risk of disease are criticized for the use of poor exposure assessment. It follows, however, from the previous discussion that crude exposure assessment cannot give rise to spurious effects; it can only mask an existing effect.

Criticism of the use of poor exposure measurement seems to be particularly common in areas in which epidemiologic research is new, and it comes mostly from experts trained in exposure assessment of the relevance for the research area in question. It is important in the communication with these experts to address the issue of nondifferential misclassification.

\section{Confounding}

It is theoretically always possible that the result of a study is explained by confounding; this assumption holds true also for randomized experiments. It is therefore common that observed relationships are discredited on the basis of suggested uncontrolled confounding. Again, this is an argument usually made not by epidemiologists but by experts in other research areas. There are two points to be made in relation to this possibility.

First, as stated before regarding the character of confounding, in order for a confounder to produce a sizeable bias, the association with both the disease and the exposure have to be substantial, and one of them even has to be strong. Hence, a relative risk on the order of, say, 2.0 or more is unlikely to be explained by con- founding, at least not by one single confounder. Nevertheless there are situations within occupational epidemiology in which potential risk factors are highly correlated. Exposure to chemical substances such as nickel and chromium, for example, may be highly correlated and thus provide for the possibility of substantial confounding.

Second, one should not criticize a study for uncontrolled confounding unless some factor which is reasonably likely to be a confounder can be presented. Until such a factor is presented, one has to draw conclusions on the basis of the best available information as advised by Hill (1). This situation means, of course, that conclusions may have to be altered as new knowledge comes along, ie, when a new risk factor has been reasonably well established, and found to occur in association with the exposure at issue.

\section{Lack of a known mechanism}

The final argument to be discussed, against the reluctance to accept observed relationships, is the lack of prior knowledge supporting the observation and in particular the lack of a known possible mechanism. In the evaluation of the evidence for a possible effect the biological plausibility is, of course, of great importance. However, an epidemiologic study has to be evaluated on its own merits. The validity of a good epidemiologic study is not compromised by the fact that basic research does not provide support for the finding, and similarly a bad study does not become any better if the result has support in a known biological mechanism.

The biological plausibility has to be taken into account when the epidemiologic evidence is pooled with information from other sources. In this pooling the lack of a known possible mechanism should be given substantial weight. It is a matter of judgment, however, how heavy this weight should be. The lack of knowledge about a potential mechanism should not block new lines of research from being pursued, and, if the epidemiologic evidence is overwhelming, even firm conclusions will have to be accepted without the mechanism being fully understood.

In this respect the relationship between smoking and lung cancer may serve as an example. When studies on smoking and lung cancer were first published, very little was known about the possible mechanisms, and, indeed, the knowledge is still limited. Yet, for some considerable time, most people have considered the causal nature of this relationship justified beyond any doubt.

\section{Public health aspects}

The information available from the scientific literature is only one aspect taken into consideration when it comes to policy making in the context of public 
health decisions. It should be fully realized that not only epidemiologic evidence, but also other factors and aspects build up the background of information for preventive measures. In addition circumstances outside the realm of science contribute in a substantial way; such circumstances include the societal importance of the exposure, the number of exposed people, the seriousness of the disease involved, the practical possibilities for reducing exposure, anxiety in the society, etc. Thus the preventive activities undertaken involve political realities.

In practice, when the scientific evidence for a causeeffect relationship is uncertain, the uncertainty is often used to support the standpoint of no action. Clearly, subjective values play a role in this decision-making process, and different people do not necessarily reach the same conclusions, both depending on and regardless of their own interests.

Standards do, however, exist and do play a role in decision making. Scientific standards should be used when causal associations are being separated from noncausal ones. Most of these standards are biased towards the principle of parsimony. Events are unrelated unless strong evidence points towards the opposite. We suggest that, in the context of decisions with public health consequences, this bias should be balanced with the moral principles taken as the "benefit of the doubt." Hence the persons being subjected to a potential risk should benefit from the doubts limiting a causal interpretation. This principle, however, does not automatically translate into social action, but further elaboration on this issue is beyond the scope of the present article.

\section{Acknowledgments}

This study was supported by grant no 42.70 .05 from the Nordic Council of Ministers.

\section{References}

1. Hill AB. The environment and disease: association or causation? Proc R Soc Med 1965;58:295-300.

2. Hernberg S. "Negative" results in cohort studies - how to recognize fallacies. Scand J Work Environ Health 1981;7(suppl 4):121-6.

3. Angell M. Negative studies [Editorial]. N Engl J Med 1989;321:464-6.

4. Støttrup-Hansen E, Ahlbom A, Axelson O, Hogstedt C, Jensen UJ. Occupational epidemiologic studies with so-called negative results: no effect or no information? Stockholm: Arbetarskyddsverket, (in press). (Arbete och hälsa: in press.)

5. Popper K: The logic of scientific discovery. New York, NY: Harper and Row, 1965.

6. Greenland S. Probability versus Popper: an elaboration of the insufficiency of current Popperian approaches for epidemiologic analysis. In: Rothman KJ, ed. Causal inference. Chestnut Hill, MA: Epidemiologic Resources Inc, 1988:95-104.

7. Rothman KJ. Modern epidemiology. Boston, MA: Little, Brown and Company, 1986.

8. Walker AM. Significance tests represent consensus and standard practice. Am J Publ Health 1986;76:1033—4.

9. Walker AM. Reporting the results of epidemiologic studies. Am J Publ Health 1986;76:556-8.

10. Poole C. Beyond the confidence interval. Am J Publ Health 1987;77:195-9.

11. Flegal KM, Brownic CX, Haas JD. The effects of exposure misclassification on estimates of sedative risk. Am J Epidemiol 1986;123:736-51.

12. McMichael AJ. Standardized mortality ratios and the "healthy worker effect": scratching beneath the surface. J Occup Med 1976;18:165-8.

13. Axelson O. Aspects on confounding in occupational health epidemiology [Letter to the editor]. Scand J Work Environ Health 1978;4:98-102.

14. Miettinen OS. Theoretical epidemiology: principles of occurrence research in medicine. New York, NY: John Wiley \& Sons, 1985.

15. Greenland S. Quantitative methods in the review of epidemiologic literature. Epidemiol Rev 1987;9:1-30.

16. Teta MJ. Selection biases. Br J Ind Med 1989;46:591 - 2 .

Received for publication: 2 November 1989 